\title{
Reduced bone formation in non-steroid treated patients with rheumatoid arthritis
}

\author{
J E COMPSTON ${ }^{1}$ S VEDI, ${ }^{1}$ R W E MELLISH, ${ }^{1}$ P CROUCHER, ${ }^{1}$ AND \\ M M O'SULLIVAN ${ }^{2}$
}

From the ${ }^{1}$ Department of Pathology, University of Wales College of Medicine and the ${ }^{2}$ Department of Rheumatology, University Hospital of Wales, Cardiff

SUMmARY The cellular basis of trabecular bone loss in rheumatoid arthritis was investigated in 45 non-steroid treated patients. Mean wall thickness, an indicator of the amount of bone formed per remodelling unit, mean interstitial bone thickness, which is related to resorption depth, and the extent of trabecular surface covered by osteoid, which reflects the number of remodelling units, were assessed in iliac crest biopsy specimens. The mean wall thickness was significantly reduced in the patient group when compared with controls matched for age and sex (mean (SD) $39 \cdot 8$ (5.4) $v 51.6(9.7) \mu \mathrm{m})$. There was no significant difference between patients and controls in the mean interstitial bone thickness $(51.0(26.4) \vee 61.4(31.9) \mu \mathrm{m})$ or osteoid surface $(16.7(11.4) v 21.0$ $(10 \cdot 5) \%)$. These results show that reduced bone formation at the remodelling unit level is the predominant mechanism of bone loss in rheumatoid arthritis.

An increased prevalence of generalised osteoporosis has been reported in patients with rheumatoid arthritis, ${ }^{1-6}$ though it is uncertain whether this is due to the disease itself or to related factors such as steroid treatment ${ }^{3478}$ and inactivity. ${ }^{4910}$ Several studies have shown a reduced trabecular bone volume in iliac crest bone obtained from patients with rheumatoid arthritis, ${ }^{11-13}$ and we have recently demonstrated significant trabecular thinning in a group of such patients who had not received steroid treatment. ${ }^{13}$ The cellular basis of this bone loss has not been established; most kinetic and biochemical studies have indicated that bone formation is decreased, ${ }^{14-18}$ though others have produced evidence for increased bone turnover. ${ }^{19}{ }^{20}$ Histomorphometric data in patients with rheumatoid arthritis are scarce, but one detailed study of cortical bone in seven patients treated with salicylate reported evidence consistent with decreased bone formation. ${ }^{21}$

Bone remodelling in trabecular bone occurs at discrete sites on the trabecular bone surface, old bone tissue first being removed by resorption and then replaced by formation. ${ }^{22}$ The amount of bone formed in each of these units (basic multicellular unit) reflects bone formation at the cellular level and

Accepted for publication 19 October 1988.

Correspondence to Dr J E Compston, Department of Pathology, University of Wales College of Medicine, Heath Park, Cardiff CF4 4XN. can be assessed histomorphometrically by measuring the mean wall thickness (Fig. 1). ${ }^{23}$ In this study we report for the first time values for mean wall thickness in iliac crest trabecular bone in a group of non-steroid treated patients with rheumatoid arthritis.

\section{Patients and methods}

PATIENTS

Forty five patients (31 female, 14 male) with definite or classical rheumatoid arthritis, who had been enrolled into a trial of second line drug treatment, were studied. The mean age of all patients was 53 years (range 34-71). The duration of disease was 1-25 years (median 4), and none had received systemic steroid treatment at any time. Most patients were taking one or more of a variety of nonsteroidal anti-inflammatory drugs; in addition, all were receiving second line drug treatment (penicillamine, hydroxychloroquine, auranofin, or intramuscular sodium aurothiomalate). Patients who were housebound or restricted to a wheelchair were excluded from the study. Written informed consent was obtained from each patient, and permission for the study was granted by the local ethical committee. Disability was assessed by the Steinbroker ${ }^{24}$ and London ${ }^{25}$ methods.

CONTROLS

Control values for the indices measured in bone 


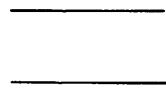

(a)

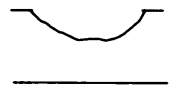

(b)

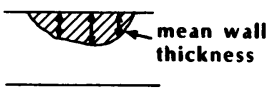

(c)
Fig. 1 Schematic representation of trabecular bone remodelling showing (a) a length of trabecula. Bone resorption by osteoclasts produces a resorption cavity (b) along the mineralised bone surface. Bone resorption is followed by bone formation, the cavity being filled in by osteoid, which then mineralises to form bone. (c) The mean thickness of the completed unit, which represents the amount of bone formed, is known as the mean wall thickness.

were obtained from 41 healthy subjects ( 28 female, 13 male) matched for age and sex, aged $31-80$ years (mean 52). These biopsy specimens were obtained during general anaesthesia for a minor surgical procedure. Details of these subjects have been published elsewhere ${ }^{26}$; none had any history of gastrointestinal disease or surgery, liver disease, renal disease, or metabolic bone disease, and none was taking anticonvulsants, corticosteroids, or any drug known to interfere with vitamin D metabolism. The study was approved by the local ethical committee.

BONE HISTOMORPHOMETRY

Transiliac biopsy specimens were obtained one inch below and behind the anterior superior iliac spine using a 6 or $8 \mathrm{~mm}$ internal diameter trephine. Undecalcified sections $(8 \mu \mathrm{m})$ were stained by the von Kossa technique or solochrome cyanin $\mathbf{R}$ or by haematoxylin and eosin after decalcification of sections in edetic acid. Mean wall thickness was measured as the distance between the cement line and the mineralised bone surface of completed walls without resorption lacunae or osteoid seams. Measurements were made under polarised light on solochrome cyanin $\mathbf{R}$ or haematoxylin and eosin stained sections and four equidistant measurements

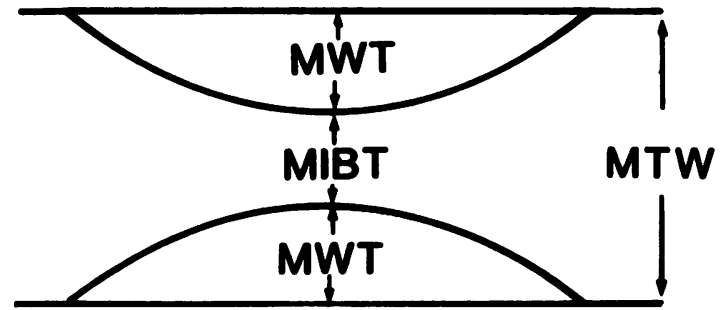

Fig. 2 Schematic diagram of part of a trabecula. The mean trabecular width (MTW) is determined by the mean wall thickness (MWT) and the mean interstitial bone thickness $(M I B T)$, the relation between these three being expressed as $M T W=(2 \times M W T+M I B T)$. were made for each packet at $\times 100$ magnification keeping the micrometer perpendicular to the sur $\overrightarrow{\overrightarrow{\vec{O}}}$ face. A minimum of 30 packets was measured from? each biopsy specimen from four or more sections. II a study of six biopsy specimens it was shown that similar values for mean wall thickness were obtaine $\bar{b}$ using either haematoxylin and eosin or solochrome cyanin R (47.6 (7.2) v 51.2 (8.4) $\mu \mathrm{m} ; \mathrm{p}=\mathrm{NS})$. Then mean interstitial bone thickness was calculated as the mean trabecular width $-2 \times$ mean wall thick $=$ ness $^{27}$ (Fig. 2) using values obtained previously fo mean trabecular width. ${ }^{13}$

As a constant mean interstitial bone thicknes $\stackrel{\varrho}{\Omega}$ may be associated with a constant or decreasedo resorption depth, and a decreased mean interstitiaps bone thickness with an increased, constant, obै decreased resorption depth ${ }^{28}$ we also calculated the approximate mean resorption depth in patients and controls. The mean resorption depth in controls was assumed to be the same as the mean wall thickness this is probably a slight underestimate of the true resorption depth, but in one study of normate subjects no differences were found between directlye measured wall thickness and resorption depth. ${ }^{29}$ In addition, it was assumed that the mean trabecular

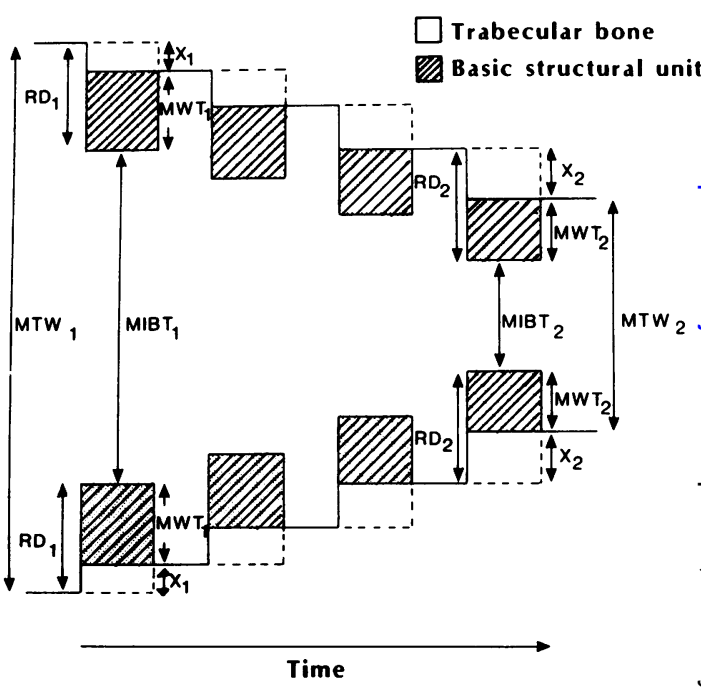

Fig. 3 Diagram to show how resorption depth is calculated. $R D$, represents resorption depth before the onset of rheumatoid arthritis and is assumed to be quantitatively similar to mean wall thickness $\left(M W T_{1}\right) . R D_{2}$ represents resorption depth in the patient group and is equal to $M W T_{2}+\left(M T W_{1}-M T W_{2}\right) / 2$, where $M T W_{1}$ is the mean trabecular width before the onset of rheumatoid arthritis and $\mathrm{MTW}_{2}$ is the mean trabecular width in the patient group. In this model it is assumed that values in the patients were similar to those of controls before the onset of their illness. 
width in the patient group before the development of rheumatoid arthritis was similar to that found in normal controls. In the patient group resorption depth was calculated as follows:

$$
\text { Resorption depth }(\mu \mathrm{m})=\mathrm{MWT}_{\mathrm{RA}}+\frac{\left(\mathrm{MTW}_{\mathrm{c}}-\mathrm{MTW}_{\mathrm{RA}}\right)}{2}
$$

where $\mathrm{MWT}_{\mathrm{RA}}=$ mean wall thickness in patients; $\mathrm{MTW}_{\mathrm{c}}=$ mean trabecular width in controls; MTW $_{\text {RA }}=$ mean trabecular width in patients (Fig. 3).

Osteoid surface (percentage total trabecular surface) was calculated from osteoid volume and mean osteoid seam width, measured on the Ibas II image analyser in a minimum of 40 fields from three or more von Kossa stained sections at a magnification of $\times 207$. Osteoid surface was calculated as follows:

$$
\text { Osteoid surface }(\%)=\frac{\text { osteoid volume }}{\text { mean osteoid seam width }(\mu \mathrm{m})} \times 100
$$

where osteoid volume is expressed as a percentage of total trabecular bone volume.

\section{STA TISTICS}

Differences between patients and controls were examined using a two tailed unpaired Student's $t$ test. Correlations between variables were examined by linear regression analysis or one way analysis of variance.

\section{Results}

The mean (SD) wall thickness in the whole group of patients was significantly lower than that in controls matched for age and sex $(39.8(5.4) \nu 51.6(9.7) \mu \mathrm{m}$, $\mathrm{p}<0.001)$. When patients were divided into men and women (Fig. 4) this significant difference was maintained in each sex, the mean values in female patients and controls being $38.5(5.3)$ and 52.2 $(11.0) \mu \mathrm{m}$ respectively $(\mathrm{p}<0.001)$ and in men 42.9 $(4.3)$ and $50.4(6.3) \mu \mathrm{m}$ respectively $(\mathrm{p}<0.002)$. The mean osteoid surface in the patients was 16.7 $(11.4) \%$, the control value being $21.0(10.5) \%$ $(\mathrm{P}=\mathrm{NS})$. The mean trabecular width in the patients was lower than that in controls $(131.1(23.6) v 159.2$ $(19.8) \mu \mathrm{m}, \mathrm{p}<0.001)$; details of these findings have been published previously. ${ }^{13}$

The mean interstitial bone thickness in all patients $(51 \cdot 0(26 \cdot 4) \mu \mathrm{m})$ did not differ significantly from that in the control group $(61.4(31.9) \mu \mathrm{m})$. In female patients, however, there was a significant decrease in mean interstitial bone thickness when compared with controls $(46.3(21.6) v 65.3(36.0) \mu \mathrm{m}, \mathrm{p}<0.05)$ (Fig. 5). In men, the mean values obtained in patients and controls were similar $(61.5(33.3) v 55.0$ (23.6) $\mu \mathrm{m}, \mathrm{p}=\mathrm{NS}$ ).

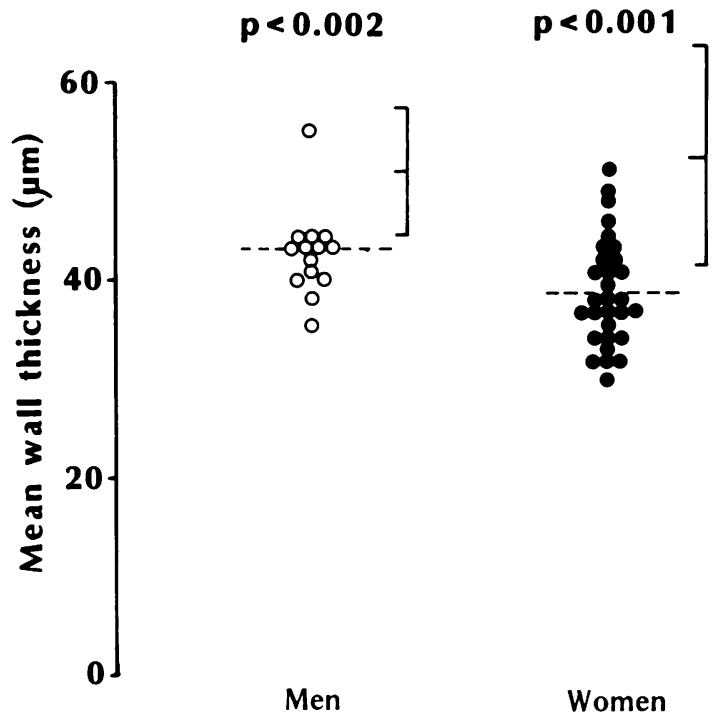

Fig. 4 Mean wall thickness in male and female patients with rheumatoid arthritis. The interrupted horizontal lines show the mean values. The mean (SD) in controls matched for age and sex is shown by the vertical continuous lines.

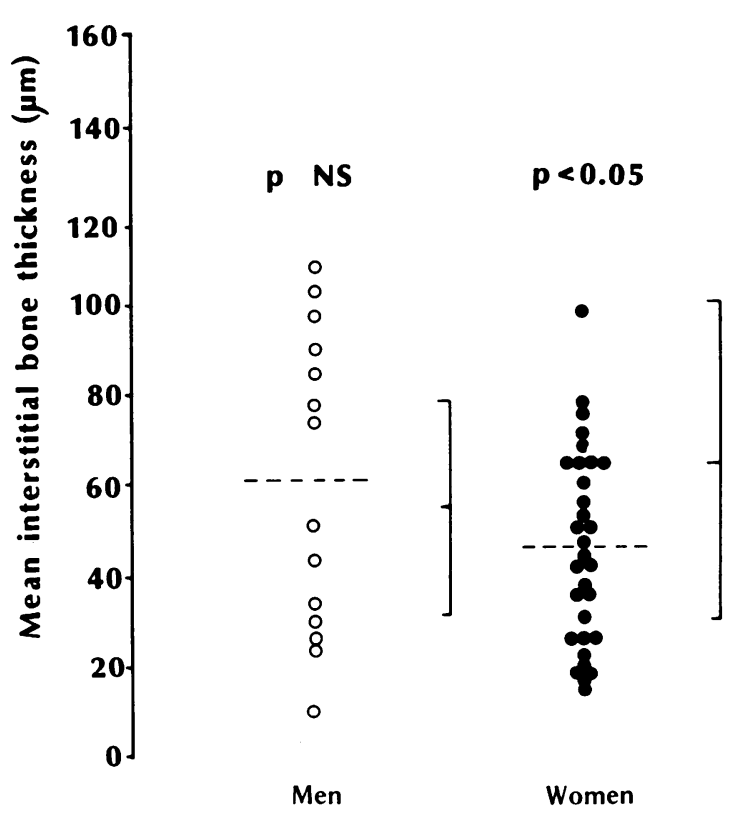

Fig. 5 Mean interstitial bone thickness in male and female patients with rheumatoid arthritis. The interrupted horizontal lines show the mean values. The mean (SD) in controls matched for age and sex is shown by the vertical continuous lines. 
Calculation of the approximate resorption depth (see 'Patients and methods') showed a mean resorption depth of $59.0 \mu \mathrm{m}$ in female patients and $52.2 \mu \mathrm{m}$ in female controls. In men the corresponding figures were 47.4 and $50.4 \mu \mathrm{m}$. When all patients were grouped together the mean calculated resorption depth was $51.6 \mu \mathrm{m}$ compared with $54.8 \mu \mathrm{m}$ in the controls.

Linear regression analysis showed a significant positive correlation between mean wall thickness and mean trabecular width $(r=0 \cdot 281, p<0 \cdot 05)$. No significant correlation was found between mean wall thickness and duration of disease, Steinbroker or London scores.

\section{Discussion}

Our results show a highly significant decrease in the amount of bone formed at trabecular remodelling sites in non-steroid treated patients with rheumatoid arthritis, and provide the first unequivocal histomorphometric evidence of reduced bone formation in these patients. These results are consistent with our earlier demonstration of trabecular thinning in the same group of patients ${ }^{13}$ as bone loss due predominantly to reduced bone formation would tend to result in trabecular thinning, whereas bone loss associated with increased bone turnover with or without an increase in resorption depth would decrease the connectedness of trabecular bone with less effect on trabecular width. Our findings are also compatible with those reported by Ramser et al in rib cortical bone ${ }^{21}$; they showed reduced tetracycline uptake in three of five patients and reduced osteoid surface in four of seven patients with rheumatoid arthritis treated with salicylate. The increase in surface extent of resorption, which was found in all seven patients, may also have reflected decreased formation rather than increased resorption, previously resorbed cavities not being filled by new bone because of reduced bone formation.

The pathogenesis of reduced bone formation in rheumatoid arthritis has not been established. Decreased mean wall thickness in trabecular bone has been reported in corticosteroid induced osteoporosis ${ }^{30}$ but none of the patients in the present study had received systemic steroids. Decreased bone formation has also been demonstrated in osteoporosis due to immobilisation ${ }^{31}$; none of our patients was housebound or restricted to a wheelchair, however, and, moreover, no correlation was found between mean wall thickness and indices of functional ability. Nevertheless, some effect of reduced mobility on bone formation in these patients cannot be excluded as functional assessments made at one point in time do not reflect accurately either disease duration or its past activity; in some studies? disease duration and activity have been shown to correlate with measurements of bone loss. ${ }^{168932} 339$ Other possible factors which may contribute to bone loss in rheumatoid arthritis include non-steroida anti-inflammatory compounds and drugs used in second line treatment; the effects, if any, of thesed agents on bone remodelling have not been studied.

The values obtained for mean interstitial bonethickness in this study showed a significantly lower mean value in female patients than in controls $\overrightarrow{\vec{\omega}}$ matched for age and sex but no significant differenceo in male patients. Although the mean interstitial bone thickness is related to resorption depth, this is not a simple inverse relation as the mean interstitial $0_{\infty}^{\infty}$ bone thickness is affected not only by resorption: depth but also by changes in the mean wallew thickness. ${ }^{28}$ Thus, depending on concurrent changeso in mean wall thickness, a decreased mean interstitial bone thickness may be associated with a decreased,, constant, or increased resorption depth, whereas a normal mean interstitial bone thickness could occur with either a constant or decreased resorption $\infty_{0}$ depth. Calculation of the resorption depth from. mean trabecular width and mean wall thicknesso indicated, however, that resorption depth was closes to normal in both male and female patients, thuso excluding the possibility that increased resorptiono depth may also contribute to bone loss in rheuma- $-\mathbb{D}$ toid arthritis. This finding is also consistent with our $\overrightarrow{\vec{A}}$ earlier demonstration of trabecular thinning in these 3 patients ${ }^{13}$ as increased resorption depth together? with a reduction in mean wall thickness wouldo rapidly lead to trabecular perforation with loss of connectedness.

In addition to reduced bone formation in indi-음 vidual remodelling units, which leads to irreversible bone loss, an increase in the activation of boneo remodelling units along the trabecular surface may? also result in trabecular bone loss, this being? potentially reversible. Some estimate of whether the $D$ number of remodelling sites is increased or not can be obtained from measurement of the extent of then trabecular bone surface covered by resorption cavities or osteoid; as discussed earlier, however, the $N$ surface extent of resorption may be increased as a result of decreased bone formation rather than because of increased activation, so that the osteoido surface is probably a more reliable indicator. In this study the osteoid surface was within normal limits in ? all patients and the mean value did not differ significantly from that of the control group. Similaro results have been reported in two other studies of iliac crest bone histomorphometry, ${ }^{11}{ }^{12}$ whereas ${ }_{\square}^{\mathbb{D}}$ osteoid surface was found to be reduced in ribo cortical bone in the study of Ramser et al. ${ }^{21}$ Taken 
together, these studies thus indicate that the number of remodelling sites which are activated along the trabecular bone surface is not increased in rheumatoid arthritis and that decreased bone formation at the remodelling unit level therefore represents the main mechanism of bone loss in these patients.

The clinical significance of bone loss in rheumatoid arthritis has not been clearly established. There is some evidence that fracture rate is increased in patients treated with corticosteroids, but no such evidence exists for those not receiving steroids. ${ }^{34} 35$ The role for routine prophylaxis against bone loss in these patients is therefore unclear; however, the demonstration that bone loss is predominantly due to reduced bone formation indicates that where treatment is required a drug which stimulates osteoblasts, such as sodium fluoride, may be most appropriate.

We are grateful to the Arthritis and Rheumatism Council, the Welsh Office, and Sandoz Pharmaceuticals for generous financial support. We also thank Dr J D Jessop for allowing us to study patients enrolled in the second line drug treatment trial.

\section{References}

1 McConkey B, Fraser G M, Bligh A S. Transparent skin and osteoporosis. Ann Rheum Dis 1965; 24: 210-23.

2 Duncan H, Frost H M, Villanueva A R, Sigler J W. The osteoporosis of rheumatoid arthritis. Arthritis Rheum 1965; 8: 943-54.

3 Saville P D, Kharmosh O. Osteoporosis of rheumatoid arthritis: influence of age, sex and corticosteroids. Arthritis Rheum 1967; 10: $423-30$.

4 Bjelle H O, Nilsson B E. Osteoporosis in rheumatoid arthritis. Calcified Tissue Research 1970; 5: 327-32.

5 Mueller M N, Jurist J M. Skeletal status in rheumatoid arthritis: a preliminary report. Arthritis Rheum 1973; 16: 66-70.

6 Kennedy A C, Lindsay R. Bone involvement in rheumatoid arthritis. Clin Rheum Dis 1977; 3: 403-20.

7 Als O S, Christiansen C, Hellensen C. Prevalence of decreased bone mass in rheumatoid arthritis. Relation to anti-inflammatory treatment. Clin Rheumatol 1984; 3: 201-8.

8 Reid D M, Kennedy N S J, Smith M A, Tothill P, Nuki G. Total body calcium in rheumatoid arthritis: effects of disease activity and corticosteroid treatment. $\mathrm{Br}$ Med $J$ 1982; 285: 330-2.

9 Hancock D A, Asiedu-Offei S, Atkinson P J, Reed G W, Wright V. Femoral bone mass in patients with rheumatoid arthritis and osteo-arthrosis. Rheumatology and Rehabilitation 1978; 17: 65-71.

10 Castillo B A, El Sallab R A, Scott J T. Physical activity, cystic erosions and osteoporosis in rheumatoid arthritis. Ann Rheum Dis 1965; 24: 522-6.

11 Ng K C, Revell P A, Beer M, Boucher B J, Cohen R D, Currey H L F. Incidence of metabolic bone disease in rheumatoid arthritis. Ann Rheum Dis 1984; 43: 370-7.

12 Wordsworth B P, Vipond S, Woods C G, Mowat A G. Metabolic bone disease among in-patients with rheumatoid arthritis. Br J Rheumatol 1984; 23: 251-7.

13 Mellish R W E, O'Sullivan M M, Garrahan N J, Compston J E. Iliac crest trabecular bone mass and structure in patients with non-steroid treated rheumatoid arthritis. Ann Rheum Dis 1987; 46: 830-6.

14 Dymling J F. Calcium kinetics in osteopenia and parathyroid disease. Acta Med Scand 1964; 175 (suppl 408): 16-18.
15 Heaney R P, Walch J J, Steffes P, Skillman T G. Periarticular bone remodelling in rheumatoid arthritis. Calcified Tissue Research 1968; 2 (suppl): 33-33B.

16 Sambrook P N, Ansell B M, Foster S, et al. Bone turnover in early rheumatoid arthritis. 1. Biochemical and kinetic indexes. Ann Rheum Dis 1985; 44: 575-9.

17 Orth R W, Weisman M H, Catherwood B D, Manolagas S C, Deftos L J. Bone loss in rheumatoid arthritis. Arthritis Rheum 1983; 26 (suppl 4): Abstr. 100.

18 Riis B J, Als O S, Christiansen C, Catherwood B D, Deftos L J. Bone turnover in rheumatoid arthritis. Calcif Tiss Int 1984; 36 (suppl 2): S4.

19 Rosenspire K C, Kennedy A C, Steinbach J, Blau M, Green F A. Investigation of the metabolic activity of bone in rheumatoid arthritis. J Rheumatol 1980; 7: 469-73.

20 Gevers G, Devos P, DeRoo M, Dequeker J. Increased levels of osteocalcin (serum bone GLA-protein) in rheumatoid arthritis. Br J Rheumatol 1986; 25: 260-2.

21 Ramser J R, Duncan H, Landeros O, Epker B, Frost H M. Measurements of bone dynamics in seven patients with salicylate-treated rheumatoid arthritis. Arthritis Rheum 1966; 9: 424-9.

22 Frost H M. Bone remodelling dynamics. Springfield, Illinois: Thomas, 1963.

23 Lips $\mathbf{P}$, Courpron $\mathbf{P}$, Meunier $\mathbf{P} \mathbf{J}$. Mean wall thickness of trabecular bone packets in the human iliac crest: changes with age. Calcified Tissue Research 1978; 26: 13-17.

24 Steinbroker O, Traeger C H, Batterman E C. Therapeutic criteria in rheumatoid arthritis. JAMA 1949; 140: 659-62.

25 Kirwan J R, Reebach J S. Using a modified Stamford health questionnaire to assess disability in UK Patients with RA [Abstract]. Proceedings of the Heberden Society AGM. London: Heberden Society, 1982: 22.

26 Vedi S, Compston J E, Webb A, Tighe J R. Histomorphometric analysis of bone biopsies from the iliac crest of normal British subjects. Bone 1982; 4: 231-6.

27 Courpron $\mathbf{P}$, Lepine $\mathbf{P}$, Arlot $\mathbf{M}$, Lips $\mathbf{P}$, Meunier $\mathbf{P} \mathbf{J}$. Mechanisms underlying the reduction with age of the mean wall thickness of trabecular basic structure unit (BSU) in human iliac bone. In: Jee W S S, Parfitt A M, eds. Bone Histomorphometry, Third International Workshop. Paris: Armour Montagu, 1980: 323-9.

28 Croucher P I, Mellish R W E, Vedi S, Garrahan N J, Compston $J$ E. The relationship between resorption depth and mean interstitial bone thickness (MIBT): age-related changes in man. Calcif Tissue Int (in press).

29 Eriksen E F. Normal and pathological remodelling of human trabecular bone: three dimensional reconstruction of the remodelling sequence in normals and in metabolic bone disease. Endocr Rev 1986; 7: 379-408.

30 Dempster D W, Arlot M A, Meunier P J. Mean wall thickness and formation periods of trabecular bone packets in corticosteroid-induced osteoporosis. Calcif Tissue Int 1983; 35: 410-7.

31 Minaire P, Meunier P, Edouard C, Bernard J, Courpron P, Bourret J. Quantitative histological data on disuse osteoporosis. Calcified Tissue Research 1974; 17: 57-73.

32 Virtama P, Helela T, Kalliomaki J L. Osteoporosis in rheumatoid arthritis. Acta Rheumatologica Scandinavica 1968; 14: 276-84.

33 Oka M, Rekonen A, Kuikka J, Antinnen J. Bone mineral density in rheumatoid arthritis measured by the gamma transmission method. Scand J Rheumatol 1975; 4: 28-32.

34 Hooyman J R, Melton L J, Nelson A M, O'Fallon W M, Riggs B L. Fractures after rheumatoid arthritis. A population-based study. Arthritis Rheum 1984; 27: 1353-61.

35 Verstraeten A, Dequeker J. Vertebral and peripheral bone mineral content and fracture incidence in post-menopausal patients with rheumatoid arthritis: effect of low dose corticosteroids. Ann Rheum Dis 1986; 45: 852-7. 